\title{
LINEAR VIBROMOTOR-ACTUATED MICROMACHINED MICROREFLECTOR FOR INTEGRATED OPTICAL SYSTEMS
}

\author{
Michael J. Daneman, Norman C. Tien, Olav Solgaard, Kam Y. Lau, Richard S. Muller \\ University of Califomia at Berkeley \\ 211-70 Cory Hall, \#1772 \\ Berkeley, CA 94720-1772
}

\begin{abstract}
We present a linear vibromotor-driven micromachined microreflector designed for integrated microphotonic applications. This device has two degrees of freedom, with an angular travel range of over $90^{\circ}$ and a translational travel range of $60 \mu \mathrm{m}$. The precision and speed of the microreflector are characterized, and it's application to beam steering and fiber coupling systems is demonstrated.

\section{INTRODUCTION}

The growing optical communications and measurement market requires low-cost, high-performance optoelectronic modules such as laser-to-fiber couplers, tunable lasers, scanners, interferometers, etc. Unlike integrated electronic circuits, integrated optical systems require precise alignment of components. A misalignment of less than $1 \mu \mathrm{m}$ can dramatically decrease system performance. While silicon-optical-bench (SOB) technology provides for hybrid integration of semiconductor lasers, lenses, and optical fibers on a silicon chip, it is typically limited to $\pm 1 \mu \mathrm{m}$ alignment tolerances (without external adjustment). Another limitation is the lack of on-chip actuated optical components (such as mirrors, gratings, lenses, etc.) As a result, $\mathrm{SOB}$ technology applications have been limited to simple systems with no more than three or four components. To overcome these limitations a micromachined free-space optical platform has been proposed [1,2]. By combining micromachined movable optical components with lasers, lenses, and fibers on a silicon substrate, we can produce complex self-aligning optical systems on a chip. In order to function in a self-contained optical module, the micromachined components must have sufficient precision and, where required, must have on-chip actuation. In this paper, we present an actuated micromachined microreflector with two degrees of freedom and on-chip resonant electrostatically driven impact actuators.
\end{abstract}

\section{DESIGN}

The actuated microreflector (Figure 1a) was designed for laser-to-fiber coupling and external-cavity-laser applications. It consists of a polysilicon mirror plate hinged to a support beam. Each of these is, in turn, hinged to a vibromotor-actuated slider. Microhinge technology [3] allows the joints to rotate out of the plane of the substrate to achieve large aspect ratios. Commonmode actuation of the sliders results in translational motion, while differential slider motion produces out-of-plane mirror rotation. These motions permit the microreflector to position an optical beam in two dimensions.

Each of the two sliders is actuated with an integrated microvibromotor (Figure 1b). The vibromotor consists of four electrostatic-comb resonators with attached impact arms driving a

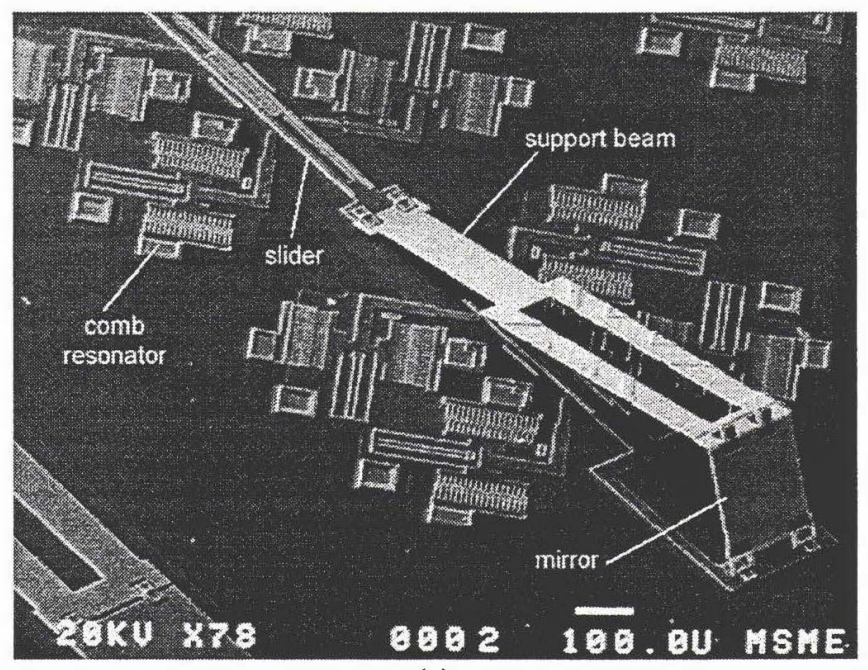

(a)

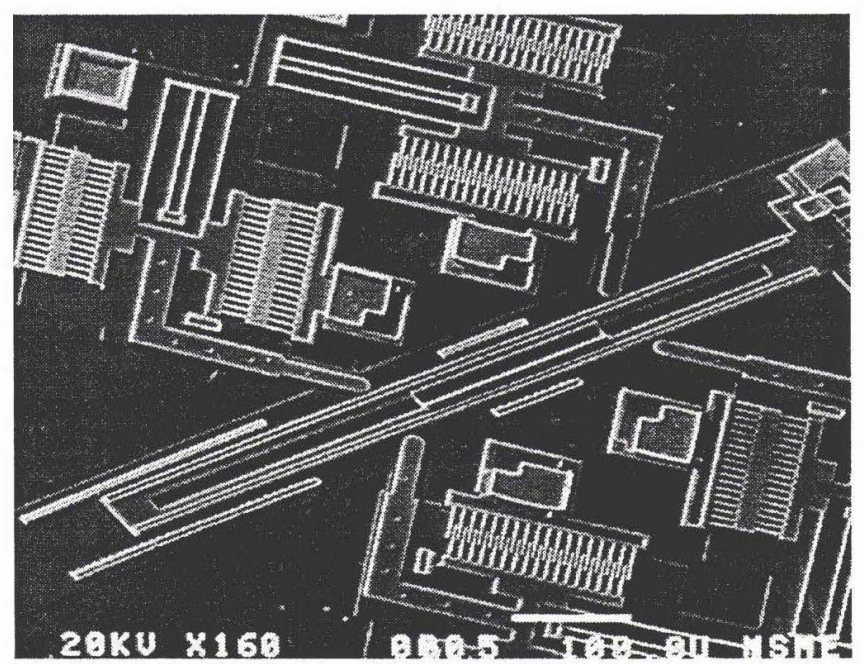

(b)

Figure 1. (a) Scanning electron micrograph of a released and assembled actuated microreflector. (b) A close up view of the integrated vibromotor actuator.

slider through oblique impact. To balance the forces, two opposing impacters are used for each direction of travel. The resonator is a capacitively driven mass anchored to the substrate through a folded beam flexure. The spring constant of the flexure determines the resonant frequency and travel range of the resonator. The force exerted by the comb drive is proportional to the square of the applied voltage 


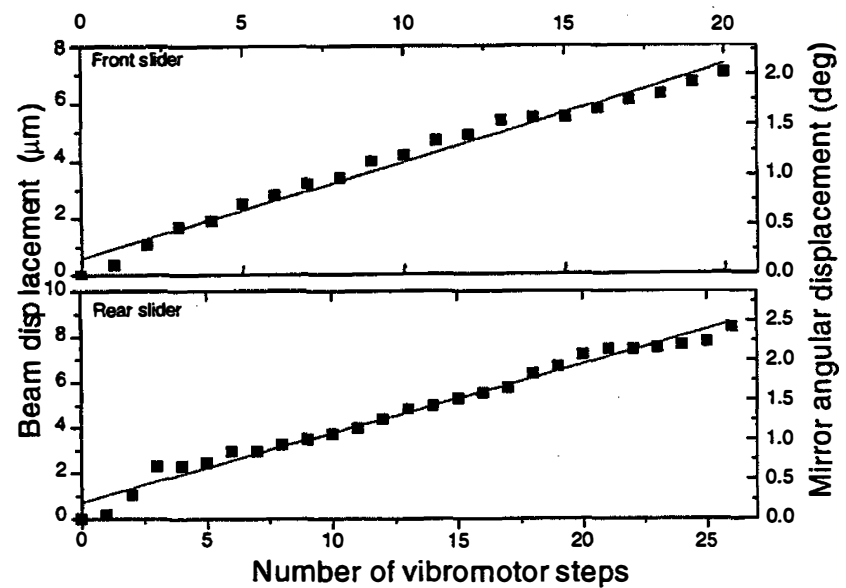

(a)

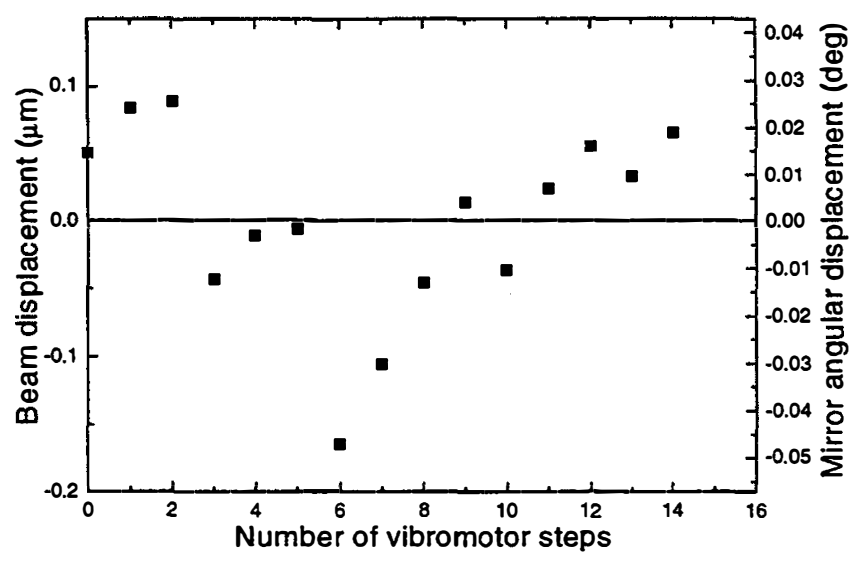

(b)

Figure 2. Vertical (a) and horizontal (b) microreflector precision. (a) The vertical position data show standard deviations of $0.32 \mu \mathrm{m}$ and $0.6 \mu \mathrm{m}$ (for the front and rear sliders respectively) from a linear response. (b) The horizontal beam position has an average $0.05 \mu \mathrm{m}$ of in-plane wobble.

$$
F=\frac{1}{2} \frac{\partial C}{\partial x} V^{2}
$$

where $V$ typically has both dc and ac components. This quadratic response produces a primary frequency driving term proportional to the product of the dc and ac voltages, effectively linearizing the resonator and increasing the impact force. The comb structures are driven at their resonance frequency (roughly $7.5-8.5 \mathrm{kHz}$ ) thereby achieving an amplification of the electrostatic force by the resonator quality factor (typically 30 to 100 in air $[4,5])$. Since energy is transferred to the slider only during impact (typically lasting only a few microseconds), the impacters can deliver short-duration forces that are large enough to overcome static friction in the sliders and hinges. Due to the damping (primarily due to airflow $[4,5]$ ) in the comb structure, the resonators require a few initial cycles to build up sufficient amplitude and momentum for impact. Therefore, in air, slider motion is observed only after three or more voltage cycles. The number of initiation cycles depends on the ambient atmosphere, and will decrease at lower pressures. When driven with a freerunning resonant oscillation, the slider reaches a maximum

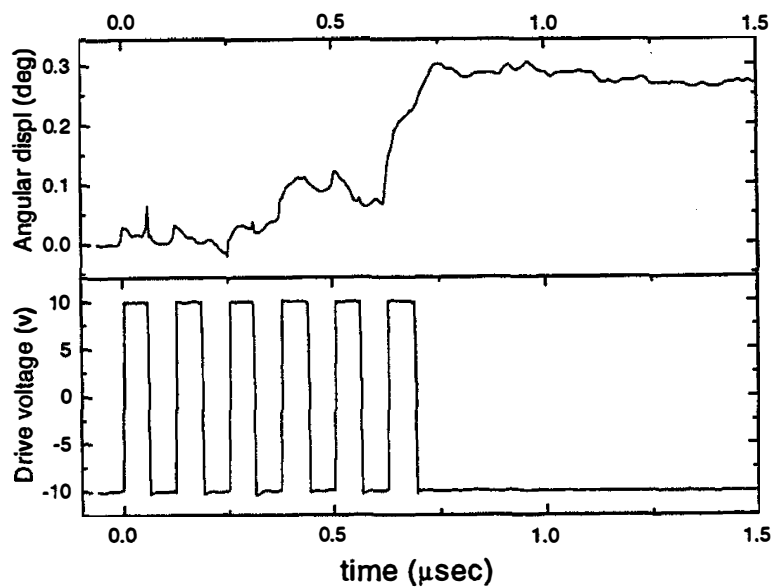

Figure 3. Time domain response of the microreflector to a square wave drive voltage. An impact is first achieved during the third cycle at which point the mirror angle begins to change. The motion concludes shortly after the last cycle resulting in a total angular displacement of about $0.3^{\circ}$.

velocity of over $1 \mathrm{~mm} / \mathrm{s}$. Slider velocity can be conrolled by driving the comb drives with gated bursts of 4 to 5 cycles of the resonant waveform. Once the slider is in position, it is kept in place by static friction until further actuation. Similar structures were previously subjected to shock and vibration tests and showed no detectable slider motion at forces up to $500 \mathrm{G}$ 's [1].

\section{FABRICATION}

The actuated microreflector was fabricated on a silicon substrate using silicon-surface micromachining technology. The fabrication process is described in detail in $[1,8]$. An $n+$ polysilicon layer defines a ground plane. Three additional polysilicon structural layers are used to define the comb-drives, sliders, hinges, and mirror beams. Phosphorous doped silicon dioxide is used for sacrificial spacer layers between polysilicon layers. A special pre-release etch in 5:1 HF followed by a vigorous rinse is used to eliminate stringers. Then the structure is released for 10 minutes in concentrated $\mathrm{HF}$ to dissolve the oxide and dried using a critical-point $\mathrm{CO}_{2}$ drier [7] to avoid stiction. A $400 \AA$ gold layer is evaporated onto mirror surface to increase reflectivity.

\section{CHARACTERIZATION}

Characterization of the vibromotor alone has been described in a previous publication and has shown the slider motion to have a step resolution of less than $0.3 \mu \mathrm{m}$ [8]. In this paper, vibromotor performance in an integrated microreflector system (Figure 1) is characterized. To measure the precision of the actuated microreflector, a $\mathrm{HeNe}$ laser is reflected from the micromirror surface onto a CCD camera. As the front and rear sliders are actuated, the beam position along each of the two axes is measured on the CCD and extrapolated to a location $200 \mu \mathrm{m}$ in front of the mirror (where a fiber would typically be positioned for a laser-to-fiber coupling application). The standard deviation of the vertical beam position data from a linear response depends on the selected slider step size. For the front slider this deviation is roughly equal to the step size, while for the rear slider the deviation exceeds the step size by about $50-60 \%$. The selected average step sizes of $0.35 \mu \mathrm{m}$ for the front slider and $0.42 \mu \mathrm{m}$ for 


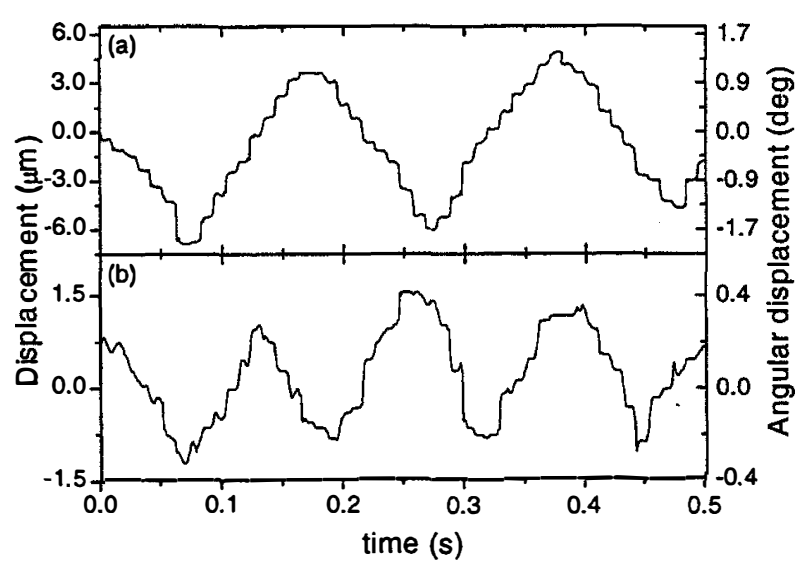

Figure 4. Beam scanning with the actuated micro-reflector. In (a) a $40 \mathrm{~V} \mathrm{dc}$ offset was used, resulting in 0.7-1.1 $\mu \mathrm{m}$ step size on the plane $200 \mu \mathrm{m}$ from the micromirror, while in (b) a $38 \mathrm{~V} \mathrm{dc}$ offset produces 0.3-0.6 $\mu \mathrm{m}$ steps.

the rear slider produce standard deviations of $0.32 \mu \mathrm{m}$ and $0.60 \mu \mathrm{m}$ respectively (Figure $2 \mathrm{a}$ ). These deviations are due primarily to the "play" in the hinges and the wobble in the slider structure. The greater length of the rear slider results in increased wobble and leads to a greater standard deviation. The horizontal beam deviation is $0.05 \mu \mathrm{m}$ (Figure $2 \mathrm{~b}$ ) and is comparable to the $0.07 \mu \mathrm{m}$ deviation measured in externally actuated structures. This precision is sufficient for laser to single-mode fiber coupling applications where, due to lens magnification, the beam only needs to be within approximately $\pm 1 \mu \mathrm{m}$ for high coupling. However, for external cavity laser applications a higher angular precision is necessary. Since in earlier experiments a microreflector with no on-chip actuators and an alternate hub design has shown a vertical precision of $0.17 \mu \mathrm{m}$ [1], improving the design of the actuated slider should greatly improve the mirror precision.

In order to observe the actuation dynamics of the microreflector, a reflected HeNe beam was imaged on a positionsensitive detector while the angular position of the mirror was

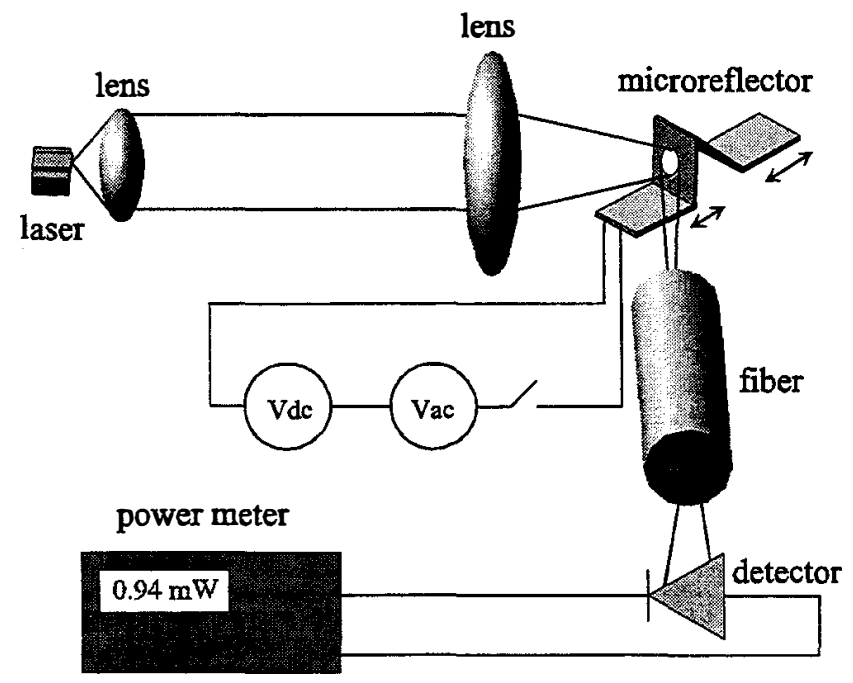

Figure 6. Experimental setup for the laser-to-fiber coupler system. The microreflector is used to optimize optical alignment into the single-mode fiber.

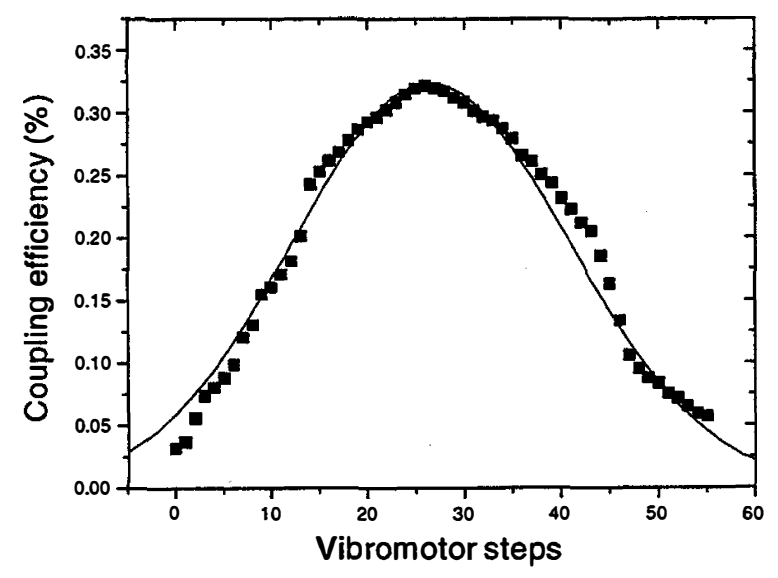

Figure 5. Coupling efficiency from semiconductor laser to singlemode fiber using the actuated micromirror. An efficiency of $32 \%$ was achieved.

swept in real time. The rear vibromotor was biased at $40 \mathrm{~V} \mathrm{dc}$ and driven with $20 \mathrm{~V}(\mathrm{p}-\mathrm{p})$ resonant $(8.2 \mathrm{kHz})$ square-wave pulses. The position of the beam and the drive voltage were then monitored on a digital oscilloscope. The resulting race is shown in Figure 3. As predicted and observed in [8], the comb structures require a few cycles ( 3 in this case) to build up sufficient energy for impact. The first significant impact occurs during the third cycle at which point the mirror angle begins to change. The mirror is further deflected during the fourth cycle; however, sufficient energy is lost by the resonator to keep the next impact from producing significant displacement. Finally, the sixth cycle provides the greatest impact, resulting in a total mirror rotation of $0.3^{\circ}$. The small back and forth motion apparent in the response is most likely due to slight deformation of the hinge joints during impact as the square pin is forced against the staple. The remaining "roughness" of the response is due to noise in the detector.

To demonstrate the use of the actuated microreflector in scanning and beam-positioning applications, the mirror was used to continuously scan a laser beam across the detector. The rear vibromotor was driven with bursts of four $20 \mathrm{~V}$ (p-p) resonant $(8.2 \mathrm{kHz})$ square-wave cycles spaced $10 \mathrm{~ms}$ apart, with a 40 or $38 \mathrm{~V} \mathrm{dc}$ offset. The resulting output (Figure 4) clearly shows the stepwise nature of the mirror motion. The average speed of the sweep can be changed by varying the spacing of the bursts. Figures $5 \mathrm{a}$ and $5 \mathrm{~b}$ also demonstrate that the size of the step itself depends on the applied voltage and can be controlled. With an average angular step size of $5 \mathrm{mrad}$, the microreflector has a maximum estimated scan rate of $10.2 \mathrm{rad} / \mathrm{s}$ or a beam speed of $2 \mathrm{~mm} / \mathrm{s}$ on a plane $200 \mu \mathrm{m}$ in front of the reflector.

Next the microreflector was used in a fiber coupler configuration to provide fine alignment between a semiconductor laser and a single-mode optical fiber ( $9 \mu \mathrm{m}$ core). The light from a standard telecommunications grade $1.3 \mu \mathrm{m}$ DFB laser was imaged by a lens system onto the fiber facet. All components were kept on separate alignment stages, and the actuated microreflector was positioned between the lens and the fiber to provide fine alignment (Figure 6). The microreflector was actuated to provide a maximum coupling efficiency of $32 \%$. Figure 5 shows the dependence of coupling efficiency on the number of rear vibromotor steps. The step size can be varied by changing the drive voltage to provide various degrees of control. 
The data show a good fit to a Gaussian curve with deviations being due to slider wobble and angular misalignment of the components. The coupling efficiency was limited to $32 \%$ in this experiment due to misalignment of the bulk components. However, efficiencies up to $45 \%$ were previously demonstrated using externally actuated devices [1,6], and, with all on-chip components and a higher placement precision, should reach $70 \%$.

\section{CONCLUSIONS}

In conclusion, we have fabricated and characterized a vibromotor actuated micromachined microreflector for use in integrated optical systems on a silicon chip. The microreflector has two degrees-of-freedom and is able to position an optical beam in two dimensions. It is designed for use in self-contained laser-to-fiber coupling modules, integrated external-cavity devices, as well as other silicon-optical-bench applications requiring precise optical alignment. The reflector is capable of positioning an optical beam a fiber with a resolution of $0.81 \mu \mathrm{m}$, and a significantly higher precision is expected in future designs. We have demonstrated repeatable beam scanning, with the scanning speed and step size determined by the applied electrical signal. We also used the microreflector for fine alignment in a laser to single-mode fiber coupler, achieving precise control of the beam and a $32 \%$ coupling efficiency. The actuated microreflector and devices like it mark an important step towards making useful and cost-effective miniaturized integrated optical system.

\section{REFERENCES}

1. O. Solgaard, M. Daneman, N.C. Tien, A. Friedberger, R.S. Muller, K.Y. Lau, "Optoelectronic packaging using silicon surface-micromachined alignment mirrors," IEEE Photonics Technology Letters, vol. 7, no. 1, 1995, pp. 41-43.

2. L.Y. Lin, S.S. Lee, K.S.J. Pister, M.C. Wu, "Micro-machined three-dimensional micro-optics for free-space optical systems," IEEE Photonics Technology Letters, vol. 6, no. 12, 1994, pp. 1445-1447.

3. K.S.J. Pister, M.W. Judy, S.R. Burgett, R.S. Fearing, "Microfabricated hinges," Sensors and Actuators (A), vol. 33, no. 3, 1992, pp. 249-256.

4. W.C. Tang T.-C. H. Nguyen, M.W. Judy, R.T. Howe, "Electrostatic-comb drive of lateral polysilicon resonators, " Sensors and Actuators, vol. A21-23, 1990, pp. 328-331.

5. Y.H. Cho, A.P. Pisano, R.T. Howe, "Viscous Damping Model for Laterally Oscillating Microstructures," Journal of Microelectromechanical Systems, vol. 3, no. 2, 1994, pp.8187.

6. M.J. Daneman, O. Solgaard, N.C. Tien, K.Y. Lau, R.S. Muller, "Integrated Laser-to-Fiber Coupling Module Using a Micromachined Alignment Mirror," Photonics Technology Letters, vol. 8, no. 3, March, 1996.

7. G.T. Mulhern, D.S. Soane, R.T. Howe, "Supercritical Carbon Dioxide Drying of Microstructures," 7th International Conference on Solid State Sensors and Actuators, 1993, pp. 296-299.
8. M.J. Daneman, N.C. Tien, O. Solgaard, A.P. Pisano, K.Y. Lau, R.S. Muller, "Linear Microvibromotor for Positioning of Optical Elements," Microelectromechanics Structures Conference, Amsterdam, The Netherlands, January 1995, pp. 55-60. 